\title{
Composition of mixed-species bird flocks in forest fragments of southern Brazil
}

\author{
Cláudia S. Brandt 1, 3; Heinrich Hasenack 1; Rudi R. Laps ${ }^{2} \&$ Sandra Maria Hartz 1 \\ 1 Programa de Pós-Graduação em Ecologia, Centro de Ecologia, Universidade Federal do Rio Grande do Sul. Avenida Bento \\ Gonçalves 9500, Caixa Postal 15007, 91501-970 Porto Alegre, Rio Grande do Sul, Brasil. \\ ${ }^{2}$ Laboratório de Biologia Animal e Laboratório de Ecologia e Ornitologia, Departamento de Ciências Naturais, Universidade \\ Regional de Blumenau. Rua Antônio da Veiga 140, Victor Konder, 89012-900 Blumenau, Santa Catarina, Brasil. \\ 3 Correspondig author. E-mail: claubrandt@gmail.com
}

\begin{abstract}
The present study aimed to evaluate the composition of mixed-species bird flocks in a fragmented landscape of the Atlantic Forest in southern Brazil. We recorded 117 species in 218 mixed-species flocks. The correspondence analysis showed two distinct groups of fragments according to degree of connectivity with other fragments. The percentage of original cover surrounding a sampled fragment area up to a ratio of 2.5 and $1 \mathrm{~km}$ was the variable that presented highest correlation with the formation of different composition of mixed-species bird flocks, followed by altitude. Based on these results, we concluded that, in landscapes with low levels of fragmentation, the composition of mixed flocks reflects the local species pool. Thus, future studies comparing composition of mixed-specie flocks should concentrate their efforts in areas where some of these variables remain constant, as for example where fragments are at similar altitudes.
\end{abstract}

KEY WORDS. Atlantic Forest; bird community; ecological interactions; fragmentation; mixed-species flocks.

Forest fragmentation and its effects on biodiversity are issues frequently discussed in the literature. In contrast, the number of studies addressing the effects of habitat fragmentation on social systems and the behavior of participant individuals has received little attention, at least until recently (Telleria et al. 2001). As an attempt to fulfill this gap, new approaches that focus on the ecological interactions between species, which play an important role in maintaining the integrity of local communities (Maldonado-Coelho \& Marini 2003, SRIDHAR \& SANKAR 2008), have emerged.

Mixed flocks of birds are amongst the most common and complex interactions in forest environments (Jullien \& THIOLLAY 1998). For this reason, understanding their ecology may enhance our understanding of the biology and behavior of the participant species, and help provide information to devise conservation strategies in fragmented landscapes (MALDONADOCOElHo \& Marini 2003). An example of the latter is a study conducted in the Amazon Forest (VAN Houtan et al. 2006) showing that species that participate in mixed flocks are more sensitive to habitat fragmentation, thus spending less time in fragmented landscapes when compared with solitary species.

All over the world researchers have been following mixed flocks of birds in fragmented forests (STOUfFer \& BierregaArd 1995, Telleria et al. 2001, Pomara et al. 2003, Lee et al. 2005, VAN Houtan et al. 2006). In contrast, few studies have been conducted in the Atlantic Forest, one of most endangered and fragmented biomes in the world, now less than $10 \%$ of its original size (Maldonado-Coelho \& Marini 2000, 2003, 2004). The few studies conducted in this biome suggest that the composition of mixed bird flocks is relatively low in diversity and that participant species are those best adapted to small forest fragments or fragments with new (recent) or otherwise weakly developed forest cover (Maldonado-Coelho \& Marini 2000, 2003, 2004).

In order to fully understand the effects of forest fragmentation on mixed flocks of birds in the Atlantic Forest, however, supplementary studies on other aspects of habitat degradation are needed. Among the factors that are known to affect populations and communities in fragmented landscapes are habitat loss and the territory configuration (FAHRIG 1998, GASCON et al. 1999, Villard et al. 1999). The importance of these factors, in turn, depends on the structural characteristics of the landscape and the biological aspects of the species involved (Antongiovanni \& Metzger 2005).

Where habitat fragmentation is not too severe, the resulting fragments may not be totally isolated from the remaining forest matrix or forest patches. In such cases, species composition and biological interactions between species can be influenced by how adaptable participant species are to transition zones and to changes in the structure and microclimate of the local vegetation (Howrot \& Niemi 1996, Pires et al. 2006). The forest cover left around the fragments can also influence local communities by affecting the degree of isolation between patches and the maximum size of local populations (ANDréN 1994, Villard et al. 1999).

The present study aimed to study the mixed flocks of birds in a fragmented landscape in order to ascertain whether distinct fragment configurations, different percentages of surrounding forest cover and altitude impact the composition of these flocks.

() 2009 Sociedade Brasileira de Zoologia | www.sbzoologia.org.br | All rights reserved. 


\section{MATERIAL AND METHODS}

This study took place at the Itajaí River Basin, Santa Catarina, Brazil $\left(26^{\circ} 38^{\prime}-27^{\circ} 38^{\prime} \mathrm{S}, 50^{\circ} 13^{\prime}-48^{\circ} 21^{\prime} \mathrm{W}\right)$. The climate is humid mesothermic without seasonal water deficiency (GAPLAN 1986). The vegetation is part of the Atlantic Forest biome, and has been classified as mainly Ombrophilous Dense Forest (GAPLAN 1986).

We circumscribed our study sites according to their configuration in the following manner. First, we considered any disturbed area as a non-forest matrix (BECKER et al. 2007). Inside the limits of the region under study, non-forest matrices correspond to a mosaic of urban and rural areas, as well as habitats in their first phase of regeneration ("capoeira"). Taking this into consideration, we selected eleven forest fragments that were formerly part of the same, continuous forest, and classified them as follows: 1) massive fragment (immersed in a massive forest); 2) intermediate fragment (limited by a nonforest matrix but maintaining a continuous link with the forest matrix); 3) isolated fragment (totally limited by non-forest matrices). Our study sites were separated by an average of 19.36 $\mathrm{km}(2.2-45.84 \mathrm{~km})$ from each other.

In addition to the configuration of the study sites, we also took into consideration the percentage of forest cover in the surrounding areas, regardless of whether they bear a direct connection with the study sites or not. Forest cover was evaluated based on an satellite image taken on November 27, 2005 by CBERS-INPA (Satélite Sino-Brasileiro de Recursos Terrestres Instituto Nacional de Pesquisas Espaciais) and classified into two categories with the help of the computer software Idrisi v. 15.01 (Andes) from 2006 (EASTMAN 2006): 1) original vegetation present; 2) original vegetation not present. Next, using the same program, we circumscribed circular areas (buffers) around each study site and superimposed them on the image classified according to the categories defined above. Because the forest cover may vary according with the scale used, thus influencing the results, we used three different buffer sizes, $1.0 \mathrm{~km}\left(3.14 \mathrm{~km}^{2}\right) ; 2.5 \mathrm{~km}$ $\left(19.63 \mathrm{~km}^{2}\right)$; and $5.0 \mathrm{~km}\left(78.53 \mathrm{~km}^{2}\right)$ (Fig. 1).

Sampling was conducted between May-Aug in order to avoid the reproductive season of most local bird species. In order to minimize variables that influence the composition of mixed bands, the structure of the vegetation within fragments was standardized by including only fragments composed mainly by secondary vegetation in the middle stage of plant succession (KLEIN 1980). Because most of the vegetation in the region under study grows on slight to steep slopes, we were not able to standardize the altitude. Because this variable may influence the composition of mixed bands (Kotagama \& Goodale 2004), we recorded the maximum altitude of each sampled area (Tab. I).

Each fragment area was sampled during a period of four consecutive days. Observations were conducted between 7:0010:30 am and 2:00-5:30 pm, totaling 286 hours of effort in each area.

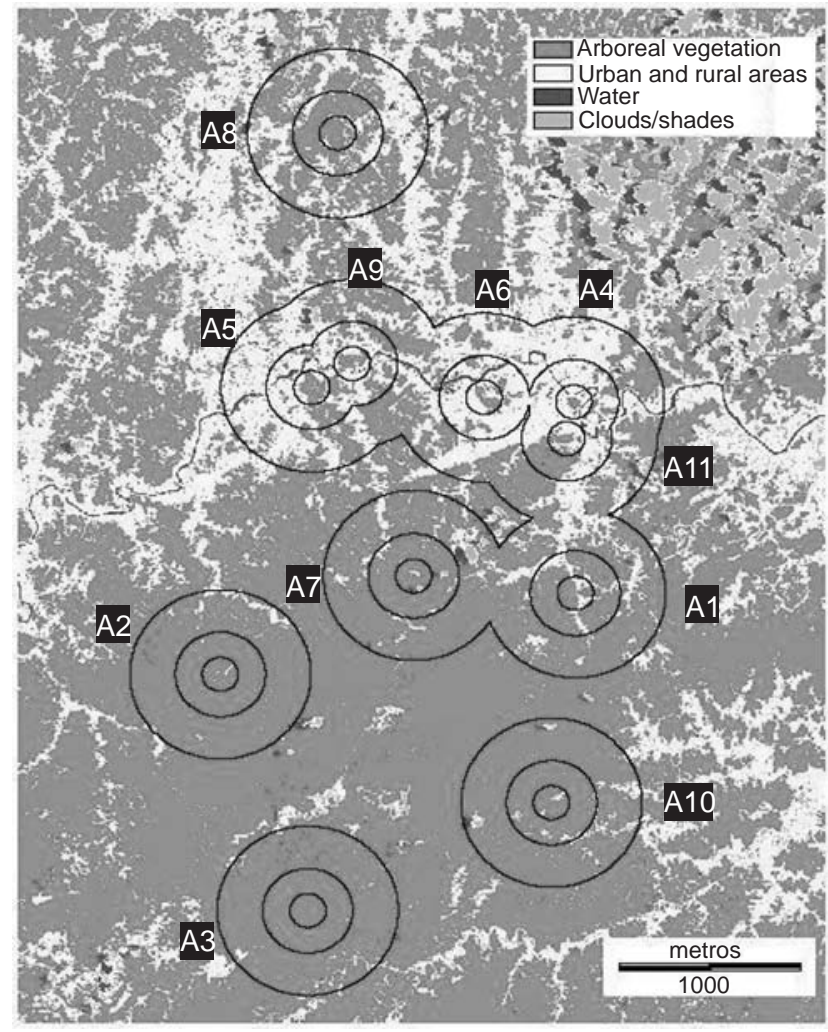

Figure 1. Atlantic Forest remnants of the hydrographic bacin of the Itajaí River, Santa Catarina, surveyed in this study. The circles indicate area buffers. The image was made available by the Instituto de Pesquisas Espaciais-INPE.

Mixed-species flocks are defined after Sтотz (1993) as associations between two or more species moving in the same direction for at least five minutes. During our surveying efforts we followed pre-existing paths, trying not to take the same path twice in order to avoid encountering a mixed flock more than once in the same fragment area. We observed each band for fifteen minutes and walked away from it in case the flock continued to remain visible after this period (MALDONADO-COELHO \& MARINi 2004).

We used correspondence analysis (CA) to evaluate similarities in species composition between mixed bird flocks, with the help of the statistics package MULTIV v.2.4 (PIllar 2006). The matrix contained the presence or absence of species in each area. Bootstrap autoresampling was used to evaluate axes stability (Pillar 1999). A given species was considered responsible for the segregation of a group of fragments when its correlation with a stable axis was $\geqslant 0.5$.

Once the stability of at least one axis of the CA and the formation of distinct fragments groups with respect to species composition were confirmed, the effects of the following variables were tested: configuration in the landscape, original for- 
Table I. Description of the characteristics of the remnants of the Atlantic Forest of the hydrographic basin of the Itajaí River, Santa Catarina, evaluated for the composition of mixed-species bird flocks.

\begin{tabular}{|c|c|c|c|c|c|c|c|}
\hline \multirow{2}{*}{ Locality name } & \multirow{2}{*}{ Code } & \multirow{2}{*}{ Configuration } & \multirow{2}{*}{ Area (ha) } & \multicolumn{3}{|c|}{ Forest cover buffer ratio (\%) } & \multirow{2}{*}{ Max. altitude $(\mathrm{m})$} \\
\hline & & & & $1 \mathrm{~km}$ & $2.5 \mathrm{~km}$ & $5 \mathrm{~km}$ & \\
\hline RPPN Bugerkopf & $\mathrm{A} 1$ & Intermediate & 57,000 & 74.0 & 82.0 & 79.0 & 528 \\
\hline Encano Alto & $\mathrm{A} 2$ & Massive & 57,000 & 92.3 & 71.3 & 88.2 & 365 \\
\hline Lageado Alto & A3 & Massive & 57,000 & 89.2 & 94.7 & 93.4 & 750 \\
\hline Morro da Banana & A4 & Intermediate & 26 & 15.1 & 16.9 & 31.0 & 155 \\
\hline Morro Geisler & A5 & Intermediate & 57,000 & 40.5 & 33.0 & 34.7 & 400 \\
\hline Associação Desportiva Cia Hering & A6 & Intermediate & 80 & 37.5 & 20.4 & 31.6 & 300 \\
\hline Warnow Alto & A7 & Massive & 57,000 & 93.4 & 98.5 & 95.6 & 600 \\
\hline Morro Azul & A8 & Intermediate & 57,000 & 77.8 & 71.3 & 55.9 & 700 \\
\hline Mulde Baixa & A9 & Intermediate & 85 & 40.5 & 37.8 & 45.5 & 50 \\
\hline Fazenda Agrião & $\mathrm{A} 10$ & Massive & 57,000 & 99.9 & 99.2 & 96.1 & 700 \\
\hline PNM São Franscisco de Assis & A11 & Intermediate & 57,000 & 38.6 & 33.2 & 39,4 & 135 \\
\hline
\end{tabular}

est cover, and maximum altitude. The statistical software R ( R Development Core Team 2007) was then used to perform a CA between these environmental variables and the scores of the stable axes for each area representing the composition of mixed bands. The results of each CA were considered significant when $\mathrm{p}$ was $>0.05$. Whenever more than one environmental variable showed significant correlation with the composition of mixed bands, a CA was conducted between them. When more than one scale of original forest cover showed significant results, only the one with a higher p-value was considered.

\section{RESULTS}

We recorded 117 species of birds in 218 mixed-species flocks (Tab. II). The number of species in each flock ranged from 18 (A4) to 71 (A2) species, with a mean of $51.09 \pm 16.66$ species/area (Tab. I).

With respect to species composition, only five species were present in all fragment areas: Dysithamnus mentalis (Temminck, 1823) ,Thamnophilidae; Xiphorhynchus fuscus (Vieillot, 1818), Dendrocolaptidae; Parula pitiayumi (Vieillot, 1817), Parulidae; Basileuterus culicivorus (Deppe, 1830), Parulidae; and Euphonia violacea (Linnaeus, 1758), Fringillidae. Furthermore, no single species was present in all mixed species flocks sampled. The different fragment areas formed two distinct groups, as shown by the stability of the first axis (\% variance $=18.10, p=0.103$ ) of the CA (Fig. 2). In other words, mixed flocks from the areas A1, A2, A4, A5, A6, A9 and A11 (Group 1) had a slightly different species composition with respect to areas A3, A7, A8 e A10 (Group 2.). In more general terms, however, the flocks were similar in all areas sampled.

Species composition of bird flocks correlated with environmental variables in various ways. An analysis of the posi- tion of these variables in relation to axis 1 of the CA (Fig. 2 and Tab. III) reveals that fragment areas in Group 1 tend to have low percentage of surrounding original forest cover, low altitudes (50 to $\sim 500 \mathrm{~m}$ ) and mainly non-forest matrix boundaries. In contrast, Group 2 is exclusively composed by fragment areas with high indexes of surrounding original forest cover, high altitudes $(\geqslant \sim 500 \mathrm{~m}$ ) and at least partial borders with the forest matrix. These tendencies are confirmed by the results of the CA, in which all environmental variables correlate positively with axis 1and, consequently, with the composition of mixed flocks (Tab. IV).

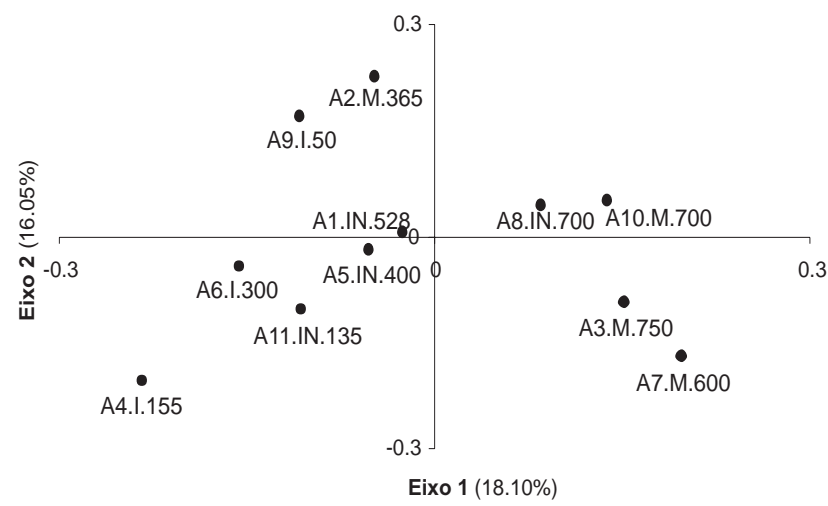

Figure 2. Correlation scores between the remnants of the Atlantic Forest of the hydrographic basin of the Itajaí River, Santa Catarina, sampled in the present study, and axis 1 of the Correspondence Analysis diagram, evaluating mixed species flock composition. The letters $\mathrm{M}, \mathrm{IN}$ and I are initials for massive fragment, intermediate fragment and isolated fragment, respectively. Numbers following the letters indicate the maximum altitude of each fragment. 
Table II. Species participating in mixed-species bird flocks sampled from remnants of the Atlantic Forest of the Hydrographic bay of the Itajaí River, Santa Catarina, between April and August, 2007.

\begin{tabular}{|c|c|c|c|c|c|c|c|c|c|c|c|}
\hline \multirow{2}{*}{ Scientific name } & \multicolumn{11}{|c|}{ Frequency in the study areas } \\
\hline & A1 & $\mathrm{A} 2$ & A3 & A4 & A5 & A6 & A7 & A8 & A9 & A10 & A11 \\
\hline \multicolumn{12}{|l|}{ Cuculidae } \\
\hline Piaya cayana (Linnaeus, 1766) & 0.24 & 0.14 & & 0.75 & & 0.13 & & & & 0.13 & 0.31 \\
\hline \multicolumn{12}{|l|}{ Trochilidae } \\
\hline Phaethornis squalidus (Temminck, 1822) & 0.08 & & & & & & & & & & \\
\hline Phaethornis eurynome (Lesson, 1832) & & 0.05 & & & & & & & & & \\
\hline Aphantochroa cirrochloris (Vieillot, 1818) & & & & & & & & 0.04 & & & \\
\hline Thalurania glaucopis (Gmelin, 1788) & 0.16 & 0.14 & & & 0.06 & 0.04 & 0.04 & 0.08 & & 0.09 & \\
\hline \multicolumn{12}{|l|}{ Trogonidae } \\
\hline Trogon surrucura (Vieillot, 1817) & 0.20 & 0.05 & & 0.25 & & 0.08 & 0.04 & 0.08 & 0.14 & 0.09 & 0.08 \\
\hline Trogon rufus (Gmelin, 1788) & 0.04 & 0.18 & & & & & & & & & \\
\hline \multicolumn{12}{|l|}{ Ramphastidae } \\
\hline Selenidera maculirostris (Lichtenstein, 1823) & & 0.05 & & & & & & & & & \\
\hline \multicolumn{12}{|l|}{ Picidae } \\
\hline Picumnus temminckii (Lafresnaye, 1845) & 0.36 & 0.09 & & & 0.31 & 0.17 & 0.25 & 0.17 & 0.29 & 0.17 & 0.15 \\
\hline Melanerpes flavifrons (Vieillot, 1818) & & 0.32 & & & 0.06 & 0.08 & & & 0.05 & & \\
\hline Veniliornis spilogaster (Wagler, 1827) & 0.28 & 0.09 & 0.06 & & 0.13 & 0.17 & 0.07 & 0.25 & 0.14 & 0.17 & 0.08 \\
\hline Piculus aurulentus (Temminck,1821) & & & 0.06 & & 0.06 & & 0.04 & 0.21 & & 0.09 & \\
\hline Dryocopus lineatus (Linnaeus, 1766) & & & & & & 0.08 & & & & & \\
\hline \multicolumn{12}{|l|}{ Thamnophilidae } \\
\hline Hypoedaleus guttatus (Vieillot, 1816) & 0.24 & 0.14 & & 0.25 & 0.06 & 0.04 & 0.14 & 0.04 & 0.05 & 0.09 & 0.08 \\
\hline Mackenziaena severa (Lichtenstein, 1823) & 0.04 & & & & & & 0.04 & & & & \\
\hline Biatas nigropectus (Lafresnaye, 1850) & & & & & & & 0.04 & & & & \\
\hline Thamnophilus caerulescens (Vieillot, 1816) & 0.04 & 0.05 & 0.06 & & & & 0.11 & 0.17 & 0.10 & 0.17 & \\
\hline Dysithamnus stictothorax (Hellmayr, 1911) & 0.08 & & & & & 0.13 & 0.07 & 0.13 & 0.43 & 0.22 & \\
\hline Dysithamnus mentalis (Temminck, 1823) & 0.16 & 0.18 & 0.17 & 0.25 & 0.38 & 0.38 & 0.25 & 0.33 & 0.38 & 0.26 & 0.08 \\
\hline Myrmotherula gularis (Spix, 1825) & 0.20 & 0.18 & 0.11 & & & & 0.04 & & & 0.04 & \\
\hline Myrmotherula unicolor (Ménétriès, 1835) & 0.20 & 0.14 & & & & & 0.07 & & 0.05 & 0.09 & \\
\hline Herpsilochmus rufimarginatus (Temminck, 1822) & 0.52 & & & & 0.19 & 0.25 & 0.11 & & & & 0.38 \\
\hline Drymophila ferruginea (Temminck, 1822) & 0.08 & 0.05 & & & & & 0.07 & 0.13 & 0.33 & & \\
\hline Drymophila rubricollis (Bertoni, 1901) & & & & & & & 0.04 & & & & \\
\hline Drymophila ochropyga (Hellmayr, 1906) & & & & & & & 0.04 & 0.04 & & & \\
\hline Drymophila malura (Temminck, 1825) & & & & & & & 0.04 & & & 0.04 & \\
\hline Terenura maculata (Wied, 1831) & 0.36 & & & & & & 0.11 & & & 0.04 & 0.08 \\
\hline Pyriglena leucoptera (Vieillot, 1818) & 0.04 & 0.09 & & & & & & 0.04 & 0.24 & 0.13 & \\
\hline Myrmeciza squamosa (Pelzeln,, 1868) & 0.04 & 0.09 & & & & & & 0.08 & 0.05 & 0.04 & \\
\hline \multicolumn{12}{|l|}{ Conopophagidae } \\
\hline Conopophaga lineata (Wied, 1831) & & & 0.06 & & & & 0.04 & 0.13 & 0.05 & 0.04 & \\
\hline Conopophaga melanops (Vieillot, 1818) & 0.20 & 0.05 & & & 0.13 & 0.08 & & 0.04 & 0.14 & 0.04 & 0.08 \\
\hline
\end{tabular}


Table II.Continued.

\begin{tabular}{|c|c|c|c|c|c|c|c|c|c|c|c|}
\hline \multirow{2}{*}{ Scientific name } & \multicolumn{11}{|c|}{ Frequency in the study areas } \\
\hline & A1 & A2 & A3 & A4 & A5 & A6 & A7 & A8 & A9 & A10 & A11 \\
\hline \multicolumn{12}{|l|}{ Rhinocryptidae } \\
\hline Psilorhamphus guttatus (Ménétriès, 1835) & 0.04 & & & & & & & & & & \\
\hline Scytalopus indigoticus (Wied, 1831) & & & & & & & & 0.04 & & & \\
\hline \multicolumn{12}{|l|}{ Formicaridae } \\
\hline Formicarius colma (Boddaert, 1783) & 0.08 & & & & & & & & & & \\
\hline \multicolumn{12}{|l|}{ Scleruridae } \\
\hline Sclerurus scansor (Menetries, 1835) & 0.04 & 0.05 & 0.06 & & 0.13 & 0.04 & 0.07 & 0.17 & & & \\
\hline \multicolumn{12}{|l|}{ Dendrocolaptidae } \\
\hline Dendrocincla turdina (Lichtenstein, 1820) & 0.16 & 0.09 & & 0.25 & 0.06 & 0.25 & 0.04 & 0.04 & 0.10 & 0.04 & 0.62 \\
\hline Sittasomus griseicapillus (Vieillot, 1818) & 0.36 & 0.55 & 0.28 & 0.5 & 0.13 & 0.04 & 0.32 & 0.25 & & 0.52 & 0.38 \\
\hline Xiphocolaptes albicollis (Vieillot, 1818) & & 0.05 & & & & & & & 0.05 & & \\
\hline Dendrocolaptes platyrostris (Spix, 1825) & 0.04 & 0.18 & & & & & & 0.04 & 0.10 & & \\
\hline Xiphorhynchus fuscus (Vieillot, 1818) & 0.32 & 0.23 & 0.28 & 0.5 & 0.25 & 0.38 & 0.29 & 0.33 & 0.43 & 0.30 & 0.77 \\
\hline Lepidocolaptes falcinellus (Cabanis \& Heine, 1859) & & & 0.06 & & & & & 0.04 & & & \\
\hline \multicolumn{12}{|l|}{ Furnariidae } \\
\hline Synallaxis ruficapilla (Vieillot, 1819) & 0.16 & 0.05 & 0.11 & & 0.19 & 0.21 & 0.32 & 0.25 & 0.48 & 0.26 & 0.08 \\
\hline Anabacerthia amaurotis (Temminck, 1823) & & 0.05 & 0.11 & & 0.06 & & 0.07 & 0.17 & & 0.30 & \\
\hline Philydor lichtensteini (Cabanis \& Heine, 1859) & 0.12 & 0.27 & 0.06 & & 0.13 & & 0.18 & 0.04 & 0.05 & 0.09 & 0.31 \\
\hline Philydor atricapillus (Wied, 1821) & 0.4 & 0.27 & 0.17 & & 0.25 & 0.21 & 0.25 & 0.17 & 0.14 & 0.39 & 0.54 \\
\hline Philydor rufum (Vieillot, 1818) & 0.08 & 0.18 & 0.22 & & & & 0.18 & 0.13 & & 0.65 & \\
\hline Anabazenops fuscus (Vieillot, 1816) & & 0.05 & & & & & 0.14 & 0.04 & & 0.30 & \\
\hline Cichlocolaptes leucophrus (Jardine \& Selby, 1830) & & 0.09 & & & & & & 0.17 & & 0.17 & \\
\hline Automolus leucophthalmus (Wied, 1821) & 0.08 & 0.05 & 0.06 & & & & 0.21 & 0.08 & & 0.26 & \\
\hline Heliobletus contaminatus (Berlepsch, 1885) & & & & & & & & & & 0.09 & \\
\hline Xenops minutus (Sparrman, 1788) & 0.12 & 0.05 & & & & 0.08 & 0.04 & & & & \\
\hline Xenops rutilans (Temminck, 1821) & 0.04 & 0.14 & 0.06 & & & 0.04 & 0.04 & 0.08 & & 0.13 & \\
\hline \multicolumn{12}{|l|}{ Tyrannidae } \\
\hline Mionectes rufiventris (Cabanis, 1846) & 0.16 & 0.18 & 0.22 & 0.25 & & 0.04 & 0.04 & & & & 0.15 \\
\hline Leptopogon amaurocephalus (Tschudi, 1846) & 0.24 & 0.41 & 0.06 & & 0.13 & 0.21 & 0.29 & 0.08 & 0.14 & 0.39 & 0.31 \\
\hline Hemitriccus obsoletus (Miranda-Ribeiro, 1906) & 0.04 & & 0.17 & & & & & & & 0.09 & \\
\hline Hemitriccus orbitatus (Wied, 1831) & 0.2 & & & & & 0.04 & & & 0.10 & & \\
\hline Poecilotriccus plumbeiceps (Lafresnaye, 1846) & & 0.05 & & & & 0.04 & 0.14 & 0.04 & 0.10 & 0.09 & \\
\hline Todirostrum poliocephalum (Wied, 1831) & & & & & & & & 0.04 & & & \\
\hline Phyllomyias virescens (Temminck, 1824) & & & & & & & & & & 0.04 & \\
\hline Camptostoma obsoletum (Temminck, 1824) & & & & & & 0.04 & & & 0.05 & & \\
\hline Plylloscartes sp. & & & & & & & & & & 0.04 & \\
\hline Phylloscartes kronei (Willis \& Oniki, 1992) & & 0.09 & & & & & & & & & \\
\hline Phylloscartes paulista (Ihering \& Ihering, 1907) & & & & & & & & & & 0.04 & \\
\hline & & & & & & & & & & & ntinue \\
\hline
\end{tabular}


Table II. Continued.

\begin{tabular}{|c|c|c|c|c|c|c|c|c|c|c|c|}
\hline \multirow{2}{*}{ Scientific name } & \multicolumn{11}{|c|}{ Frequency in the study areas } \\
\hline & A1 & $\mathrm{A} 2$ & A3 & A4 & A5 & A6 & A7 & A8 & A9 & A10 & A11 \\
\hline \multicolumn{12}{|l|}{ Tyrannidae (continued) } \\
\hline Phylloscartes sylviolus (Cabanis \& Heine, 1859) & 0.08 & & & & & & & & & & \\
\hline Myiornis auricularis (Vieillot,1818) & 0.12 & 0.36 & 0.22 & & 0.13 & 0.21 & 0.25 & 0.04 & 0.14 & 0.30 & \\
\hline Tolmomyias sulphurescens (Spix, 1825) & 0.28 & 0.23 & 0.11 & & 0.19 & 0.08 & 0.11 & 0.13 & 0.24 & 0.17 & 0.15 \\
\hline Platyrinchus mystaceus (Vieillot, 1818) & 0.08 & 0.18 & 0.28 & & 0.13 & 0.25 & 0.14 & 0.25 & 0.05 & 0.22 & \\
\hline Myiophobus fasciatus (Statius Müller, 1776) & & & & & & & 0.04 & & & & \\
\hline Contopus cinereus (Spix, 1825) & & & & & & & 0.04 & & & & \\
\hline Muscipipra vetula (Lichtenstein, 1823) & & & & & & & & & & 0.04 & \\
\hline Colonia colonus (Vieillot, 1818) & & & & & & 0.04 & 0.07 & 0.04 & & & \\
\hline Attila rufus (Vieillot, 1819) & & & & & & 0.04 & & 0.04 & & & \\
\hline \multicolumn{12}{|l|}{ Oxyruncidae } \\
\hline Oxyruncus cristatus (Swainson, 1821) & 0.04 & 0.27 & & & & & & & & & 0.08 \\
\hline \multicolumn{12}{|l|}{ Cotingidae } \\
\hline Carpornis cucullata (Swainson, 1821) & & 0.05 & & & & & 0.04 & & & & \\
\hline Lipaugus lanioides (Lesson, 1844) & & 0.05 & & & & & & & & & \\
\hline \multicolumn{12}{|l|}{ Pipridae } \\
\hline Piprites chloris (Temminck, 1822) & 0.08 & & & & & & 0.04 & & & & \\
\hline Ilicura militaris (Shaw \& Nodder, 1809) & 0.12 & 0.18 & 0.11 & & 0.06 & 0.04 & & 0.08 & & 0.09 & \\
\hline Chiroxiphia caudata (Shaw \& Nodder, 1793) & 0.20 & 0.18 & & 0.25 & 0.19 & & 0.14 & 0.08 & & 0.17 & 0.08 \\
\hline \multicolumn{12}{|l|}{ Tityridae } \\
\hline Schiffornis virescens (Lafresnaye, 1838) & 0.12 & 0.23 & 0.06 & & 0.06 & & & 0.08 & 0.05 & 0.13 & \\
\hline Pachyramphus castaneus (Jardine \& Selby, 1827) & & & 0.11 & & & & 0.07 & 0.08 & & 0.30 & \\
\hline \multicolumn{12}{|l|}{ Vireonidae } \\
\hline Cyclarhis gujanensis (Gmelin, 1789) & 0.04 & 0.05 & 0.11 & & & 0.04 & 0.07 & 0.04 & 0.05 & 0.22 & \\
\hline Hylophilus poicilotis (Temminck, 1822) & & & 0.22 & & & 0.25 & 0.07 & 0.25 & 0.29 & 0.17 & 0.31 \\
\hline \multicolumn{12}{|l|}{ Troglodytidae } \\
\hline Troglodytes musculus (Naumann, 1823) & & & & 0.25 & & & & & & & \\
\hline \multicolumn{12}{|l|}{ Polioptilidae } \\
\hline Ramphocaenus melanurus (Vieillot, 1819) & 0.08 & & & & 0.06 & 0.04 & & 0.04 & 0.05 & & \\
\hline \multicolumn{12}{|l|}{ Turdidae } \\
\hline Turdus flavipes (Vieillot, 1818) & 0.08 & 0.09 & & 0.25 & 0.31 & 0.17 & & 0.08 & & & \\
\hline Turdus rufiventris (Vieillot, 1818) & & & & 0.25 & & 0.04 & & & & & 0.15 \\
\hline Turdus amaurochalinus (Cabanis, 1850) & & & & & & & & & 0.05 & 0.04 & 0.08 \\
\hline Turdus albicollis (Vieillot, 1818) & 0.24 & 0.23 & & 0.25 & 0.06 & 0.46 & 0.04 & 0.13 & 0.29 & 0.09 & 0.38 \\
\hline \multicolumn{12}{|l|}{ Coerebidae } \\
\hline Coereba flaveola (Linnaeus, 1758) & 0.12 & & 0.06 & & 0.06 & 0.13 & 0.04 & 0.13 & 0.05 & 0.04 & 0.31 \\
\hline \multicolumn{12}{|l|}{ Thraupidae } \\
\hline Orchesticus abeillei (Lesson, 1839) & & & 0.17 & & & & & & & & \\
\hline Orthogonys chloricterus (Vieillot, 1819) & 0.04 & 0.27 & 0.06 & & & & 0.07 & & & 0.09 & \\
\hline Pyrrhocoma ruficeps (Strickland, 1844) & & & & & & & 0.07 & & & & \\
\hline Trichothraupis melanops (Vieillot, 1818) & 0.20 & 0.45 & 0.06 & & & 0.13 & 0.36 & 0.17 & 0.19 & 0.17 & 0.31 \\
\hline
\end{tabular}


Table II. Continued.

\begin{tabular}{|c|c|c|c|c|c|c|c|c|c|c|c|}
\hline \multirow{2}{*}{ Scientific name } & \multicolumn{11}{|c|}{ Frequency in the study areas } \\
\hline & A1 & A2 & A3 & A4 & A5 & A6 & A7 & A8 & A9 & A10 & A11 \\
\hline \multicolumn{12}{|l|}{ Thraupidae (continued) } \\
\hline Habia rubica (Vieillot, 1817) & 0.24 & 0.27 & 0.22 & & 0.25 & 0.21 & 0.32 & 0.25 & 0.24 & 0.48 & 0.46 \\
\hline Tachyphonus coronatus (Vieillot, 1822) & 0.16 & 0.50 & & & 0.31 & 0.67 & 0.07 & 0.13 & 0.43 & & 0.23 \\
\hline Thraupis sayaca (Linnaeus, 1766) & & 0.14 & & & & & & & 0.05 & & 0.08 \\
\hline Thraupis cyanoptera (Vieillot, 1817) & & 0.05 & & & & & & & & 0.04 & \\
\hline Thraupis ornata (Sparrman, 1789) & & 0.32 & 0.11 & & & & 0.07 & & & & \\
\hline Pipraeidea melanonota (Vieillot, 1819) & & 0.09 & & & 0.06 & & & & & 0.09 & \\
\hline Tangara seledon (Statius Müller, 1776) & 0.32 & 0.32 & 0.11 & & 0.06 & 0.04 & 0.21 & 0.13 & & 0.04 & \\
\hline Tangara cyanocephala (Statius Müller, 1776) & 0.12 & 0.32 & 0.06 & & & 0.04 & 0.18 & 0.04 & & 0.17 & \\
\hline Tangara desmaresti (Vieillot, 1819) & & & 0.06 & & & & 0.04 & & & & \\
\hline Dacnis nigripes (Pelzeln, 1856) & & 0.05 & & & & & & & & & \\
\hline Dacnis cayana (Linnaeus, 1766) & 0.08 & 0.09 & 0.06 & 0.25 & & & 0.04 & 0.04 & & 0.04 & \\
\hline Chlorophanes spiza (Linnaeus, 1758) & & 0.05 & & & & & & & & & \\
\hline Hemithraupis ruficapilla (Vieillot, 1818) & 0.28 & 0.41 & 0.06 & & 0.06 & 0.08 & 0.14 & & & 0.04 & \\
\hline \multicolumn{12}{|l|}{ Emberizidae } \\
\hline Sporophila frontalis (Verreaux 1869) & & & & & & & & & 0.10 & & \\
\hline Sporophila caerulescens (Vieillot 1823) & & & & & & & & 0.04 & & & \\
\hline Tiaris fuliginosus (Wied, 1830) & & 0.05 & & & & & & & 0.10 & & \\
\hline \multicolumn{12}{|l|}{ Cardinalidae } \\
\hline Saltator fuliginosus (Daudin, 1800) & & & & & & & & 0.08 & & & \\
\hline Saltator similis (d'Orbigny \& Lafresnaye, 1837) & & & & & & & & 0.04 & & 0.04 & \\
\hline \multicolumn{12}{|l|}{ Parulidae } \\
\hline Parula pitiayumi (Vieillot, 1817) & 0.16 & 0.50 & 0.06 & 0.5 & 0.06 & 0.13 & 0.25 & 0.13 & 0.38 & 0.13 & 0.15 \\
\hline Geothlypis aequinoctialis (Gmelin, 1789) & & 0.05 & & & & 0.04 & & & & & \\
\hline Basileuterus culicivorus (Deppe, 1830) & 0.76 & 0.82 & 0.56 & 0.75 & 0.81 & 0.71 & 0.79 & 0.75 & 0.71 & 0.70 & 0.77 \\
\hline \multicolumn{12}{|l|}{ Icteridae } \\
\hline Cacicus chrysopterus (Vigors, 1825) & & & 0.06 & & & & 0.07 & & & & \\
\hline \multicolumn{12}{|l|}{ Fringillidae } \\
\hline Euphonia violacea (Linnaeus, 1758) & 0.44 & 0.59 & 0.06 & 0.5 & 0.25 & 0.29 & 0.04 & 0.04 & 0.05 & 0.04 & 0.31 \\
\hline Euphonia pectoralis (Latham, 1801) & 0.44 & 0.36 & 0.28 & 0.25 & 0.06 & & 0.07 & 0.04 & & 0.13 & 0.08 \\
\hline Number of mixed flocks & 25 & 22 & 18 & 4 & 16 & 24 & 28 & 24 & 21 & 23 & 13 \\
\hline Number of species & 66 & 71 & 45 & 18 & 37 & 48 & 66 & 65 & 45 & 64 & 37 \\
\hline
\end{tabular}

Table III. Percent of forest cover in the surroundings of the remnants of the Atlantic Forest of the hydrographic basin of the Itajaí River, Santa Catarina, between April and August, 2007.

\begin{tabular}{|c|c|c|c|c|c|c|c|c|c|c|c|}
\hline \multirow{2}{*}{$\begin{array}{c}\text { Forest cover } \\
(\%)\end{array}$} & \multicolumn{7}{|c|}{ Area Group 1} & \multicolumn{4}{|c|}{ Area Group 2} \\
\hline & A1 & $\mathrm{A} 2$ & A4 & A5 & A6 & A9 & A11 & A3 & A7 & A8 & A10 \\
\hline Buffer $1 \mathrm{~km}$ & 74.0 & 92.3 & 15.1 & 40.5 & 37.5 & 40.5 & 99.9 & 89.2 & 93.4 & 77.8 & 99.9 \\
\hline Buffer $2.5 \mathrm{~km}$ & 82.0 & 71.3 & 16.9 & 33.0 & 20.4 & 37.8 & 99.2 & 94.7 & 98.5 & 71.3 & 99.2 \\
\hline Buffer 5 km & 79.0 & 88.2 & 31.0 & 34.7 & 31.6 & 45.5 & 39.4 & 93.4 & 95.6 & 55.9 & 96.1 \\
\hline
\end{tabular}


Table IV. Results of the Correlation Analysis between landscape configuration, native cover and maximum altitude (environmental variables) and the composition of mixed-species flocks (represented by the scores of the species with axis 1 of the Correspondence Analysis) of the remnants of the Atlantic Forest of the hydrographic basin of the Itajaí River, Santa Catarina, between April and August, 2007.

\begin{tabular}{lccc}
\multicolumn{1}{c}{ Environmental variables } & d.f. & $\mathrm{r}^{2}$ & $\mathrm{p}$ \\
\hline Landscape configuration & 9 & 0.67 & 0.001000 \\
Percentage native cover within $1 \mathrm{~km}$ ratio & 9 & 0.77 & 0.000300 \\
Percentage native cover within 2,5 km ratio & 9 & 0.84 & 0.000006 \\
Percentage native cover within 5 km ratio & 9 & 0.67 & 0.75 \\
Maximum altitude & 9 & 0.70 & 0.000400 \\
Configuration X percentage native cover within 2,5km ratio & 9 & 0.66 & 0.000700 \\
Maximum altitude X percentage native cover within 2,5km ratio & 9 & 0.46 & 0.001000 \\
Configuration X Maximum altitude & 9 & & 0 \\
\hline
\end{tabular}

The percentage of original cover surrounding a sampled fragment area up to a ratio of 2.5 and $1 \mathrm{~km}$ was the variable that presented highest correlation with the formation of Groups 1 and 2, followed by altitude. Next, configuration of the fragment area itself and the percentage of original forest cover over a ratio of approximately $5 \mathrm{~km}$ were also positively correlated with these groups. Environmental variables also correlated with one another: areas with higher altitude were surrounded and limited by more extensive original cover.

Of the 117 species in mixed-species flocks, 29 were associated with one of the two groups, i.e., showed a positive correlation $(\geqslant 0.5)$ with axis 1 of CA (Tab. V). With regards to species composition, Group 1 was composed exclusively of species that do not seem to be very sensitive to environmental changes in the region and are found anywhere in the Atlantic Forest, in addition to species that occur preferencially in forest borders, such as Turdus rufiventris (Vieillot, 1818), Turdidae; Troglodytes musculus (Naumann, 1823), Troglodytidae; and Melanerpes flavifrons (Vieillot, 1818), Picidae. In contrast, Group 2 contained species common to higher altitudes, as for example Drymophila malura (Temminck, 1825), Thamnophilidae and D. ochropyga (Hellmayr, 1906) , Thamnophilidae (found only in Group 2) and Piculus aurulentus (Temminck, 1821), Picidae; Conopophaga lineata (Wied, 1831), Conopophagidae; Philydor rufum (Vieillot, 1818), Furnariidae; and Orthogonys chloricterus (Vieillot, 1819), Thraupidae. Species that seem to be dependent on a specific micro-habitat, such as Automolus leucophthalmus (Wied, 1821), Furnariidae; Anabazenops fuscus (Vieillot, 1816), Furnariidae; and Myrmotherula gularis (Ménétriès, 1835), Thamnophilidae, were also found in Group 2.

\section{DISCUSSION}

Our results are in agreement with the expectation that the amount of original forest cover in the surroundings of forest fragment areas is important in the maintenance of several species, especially when the total coverage exceeds the $20 \%$ area (ANDRÉn 1994, FAHRIG 1998). Indeed, this variable was strongly correlated with the observed differences in species composition
Tabela V. Correlation scores between the bird species participating in mixed flocks and axis 1 of the Correspondence Analysis diagram. Only species with correlation values in relation to axis 1 $\geqslant 0.5$ are represented.

\begin{tabular}{|c|c|c|}
\hline Groups & Species correlated with axix 1 & Correlation score \\
\hline \multirow[t]{15}{*}{ Group 1} & Dendrocincla turdina & -0.778 \\
\hline & Turdus albicollis & -0.778 \\
\hline & Hypoedaleus guttatus & -0.778 \\
\hline & Turdus rufiventris & -0.726 \\
\hline & Piaya cayana & -0.678 \\
\hline & Turdus flavipes & -0.659 \\
\hline & Dysithamnus mentalis & -0.655 \\
\hline & Xiphorhynchus fuscus & -0.655 \\
\hline & Parula pitiayumi & -0.655 \\
\hline & Basileuterus culicivorus & -0.655 \\
\hline & Euphonia violacea & -0.655 \\
\hline & Trogon surrucura & -0.647 \\
\hline & Troglodytes musculus & -0.530 \\
\hline & Melanerpes flavifrons & -0.512 \\
\hline & Conopophaga melanops & -0.509 \\
\hline \multirow[t]{14}{*}{ Group 2} & Pachyramphus castaneus & 0.841 \\
\hline & Philydor rufum & 0.797 \\
\hline & Automolus leucophthalmus & 0.797 \\
\hline & Piculus aurulentus & 0.661 \\
\hline & Anabacerthia amaurotis & 0.634 \\
\hline & Myrmotherula gularis & 0.630 \\
\hline & Orthogonys chloricterus & 0.630 \\
\hline & Conopophaga lineata & 0.610 \\
\hline & Cacicus chrysopterus & 0.609 \\
\hline & Drymophila malura & 0.607 \\
\hline & Thamnophilus caerulescens & 0.603 \\
\hline & Anabazenops fuscus & 0.578 \\
\hline & Xenops rutilans & 0.539 \\
\hline & Drymophila ochropyga & 0.511 \\
\hline
\end{tabular}


of mixed flocks. This result is in agreement with VILLARD et al. (1999), who found that the percentage of forest cover and landscape configuration are important predictors of what species will be found in an area, suggesting that these two variables are correlated. An overall decrease in forest cover also determines lower connectivity between fragments, consequently decreasing the likelihood that a species that depends on the original vegetation will be found. When fragmentation happens, the original landscape and the characteristics of each species will determine how connected the various fragments will remain. For example, a study conducted in the Amazon Forest found that a distance of $30 \mathrm{~m}$ between fragments was a barrier to flock birds (Develey \& STOUfFer 2001). In contrast, mixed bird flocks in the Atlantic Forest were found migrating over distances of $100 \mathrm{~m}$ from the forest edge (Maldonado-Coelho \& Marini 2004). In any case, the surrounding matrix plays a fundamental role, as it determines permeability and consequently the ability of species and mixed bird flocks to migrate (STOufFer \& BierregaARd 1995, MaldonadoCoelho \& Marini 2000).

Territory configuration of sampled fragments was also strongly correlated with the composition of bird flocks. Forest fragments bordered by non-forest matrix (isolated or intermediate fragments) differed from areas totally immersed in a forest matrix (massive fragment). As territory configuration also showed a positive correlation with original cover around the sampled areas, it is safe to assume that these two variables had a simultaneous effect over the composition of mixed flocks. This can be attributed, at least in part, to the fact that the forest matrix can influence border effects, which are in turn correlated with the configuration of the sampled area (GASCON et al. 1999). Consequently, species that do not tolerate the matrix may also not tolerate the border, being the first ones to disappear (SEkercioglu et al. 2002). This relationship can be of particular importance for individuals that participate in mixed bands as verified by Telleria et al. (2001). Moreover, according to Stouffer \& BierregaARd (1995), species that follow mixed flocks migrate longer distances with respect to solitary birds, being more likely to encounter forest borders. As a consequence, borderline environments may cause sensitive species to disassociate from bird flock interactions and assume a solitary behavior as a strategy to survive the new circumstances. (STOUfFer \& BierregaARd 1995, Van Houtan et al. 2006). This may have been responsible for the fact that species restricted to certain habitats, as for example Anabazenops fuscus, Drymophila malura e D. ochropyga, foragers in patches of bamboos (RodRIGUEs et al. 1994, Rajão \& CerqueIra 2006), and Myrmotherula gularis, dependent on humid microclimates (SICK 1997), were only found in areas that clustered with Group 2. Species that tolerate or benefit from borderline environments, as for example Turdus rufiventris, Troglodytes musculus and Melanerpes flavifrons we incorporated in mixed bands that clustered with Group 1.

Another variable that seemed to influence the composition of mixed species flocks in this study was altitude. The few previous publications on the subject found conflicting results. For example, Kotagama \& Goodale (2004) found that the composition of mixed species flocks differed with altitude in Sri Lanka, in spite of the fact that species composition was homogenous in the areas surveyed. In contrast, SRIDHAR \& SANKAR (2008) found that the composition of mixed bands did not vary with altitude in areas with homogenous species composition. Furthermore, a study conducted by LeE et al. (2005) in Asia revealed that species that are more sensitive to altitude are less likely to participate in mixed flocks than their counterparts. Even though the distribution of birds along the altitudinal gradient of the Atltantic Forest is not completely understood (Sтотz et al. 1996, Buzzetті 2000), some studies have found a correlation between altitude and the occurrence and abundance of some species sampled in the present research. Among these are Anabacerthia amaurotis (Temminck, 1823), Furnariidae and Cacicus chrysopterus (Vigors, 1825), Icteridae (BuzzetTI 2000); P. aurulentus, D. ochropyga, O. chloricterus, M. gularis, C. lineata, A. fuscus and Xenops rutilans (Temminck, 1821), Furnariidae (Stotz et al. 1996); D. malura (RAJÃo \& Cerqueira 2006) and Philydor rufum (Aleixo 1999), all previously shown to favor high altitudes. However, because this study did not include a survey of the bird species composition and their abundance in the sampled areas, we are not able to further confirm the relationship between altitude and mixed flock composition.

Our results suggest that all variables tested correlate with mixed-species flock composition and with each other in a complex way. In order to evaluate the effects of each of these variables in isolation, future studies should concentrate their efforts in areas where some of these variables remain constant, as for example areas with fragments at similar altitudes.

The moderate fragmentation of the study area itself may be responsible for the presence of species that are sensitive to habitat degradation, independent of native cover and landscape configuration of the surroundings. In disturbed and more fragmented landscapes, mixed flocks may behave differently. For example, Maldonado-Coelho \& Marini (2004) showed that flocks in different fragments vary in complex ways, showing independent dynamics. It is also possible that the similarity in the structure of the vegetation within fragments has resulted in similar microhabitats, which can be as important to the dynamics of mixed flocks as fragmentation itself (Maldonado-Coelho \& Marini 2003, Lee et al. 2005, SRidhar \& SANKAR 2008).

Our results suggest that local mixed-species flocks are a reflection of the local bird diversity and species abundance, as advocated by previous authors (Hutto 1994, Aleixo 1997, Maldonado-Coelho \& Marini 2003, Lee et al. 2005, Ghizoni-Jr \& Azevedo 2006, SRIDHAR \& SANKAR 2008). However, future studies on landscapes with different degrees of fragmentation are needed to help us understand how the different environmental variables influence the ecology of mixed flocks in the Atlantic Forest. 


\section{ACKNOWLEDGEMENTS}

We are thank CNPq for financial support granted to Cláudia S. Brandt and Sandra M. Hartz (Process 304036/20072); Leandro Duarte for his help with statistical analysis; Pedro F. Develey, Marco A. Pizo and Andreas Kindel for their suggestions and constructive criticism; and the ornithologists Adrian E. Rupp, Carlos A. Borchardt, Daniela Fink and Gregory Thom e Silva for their help with field work.

\section{LITERATURE CITED}

Aleixo, A. 1997. Composition of mixed-species bird flocks and abundance of flocking species in a semideciduous forest if southeastern Brazil. Ararajuba 5 (1): 11-18.

Aleixo, A. 1999. Effects of selective logging on a bird community in the Brazilian Atlantic Forest. The Condor 101: 537-548.

ANDRÉn, H. 1994. Effects of habitat fragmentation on birds and mammals in landscapes with different proportions of suitable habitat: a review. Oikos 71: 355-366.

Antongiovanni, M. \& J.P. MetzGer. 2005. Influence of matrix habitats on the occurrence of insectivorous bird species in Amazonian Forest Fragments. Biological Conservation 122: 441-451.

Becker, C.G.; C. R. Fonseca; C.F.B. Haddad; R.F. Batista \& P.I. PraDO. 2007. Habitat split and the global decline of amphibians. Science 318: 1775-1777.

BuzzetTI, D.R.C. 2000. Distribuição altitudinal de aves em Angra dos Reis e Parati, sul do estado do Rio de Janeiro, Brasil. P: 131-148. In: M.A.S. Alves; J.M.C. Silva; M. VAN Sluys; H.G. Bergallo \& C.F.D. Rocha (Eds). A ornitologia no Brasil: pesquisa atual e perspectivas. Rio de Janeiro, UERJ, 352p.

Develey, P.F. \& P.C. Stouffer. 2001. Effects of roads on movements by understory birds in mixed-species flocks in Central Amazonian Brazil. Conservation Biology 15 (5): 1416-1422.

Eastman, J.R. 2006. IDRISI Andes Guide to GIS and Image Processing. Worcester, Clark Labs, 328p. Fahrig, L. 1998. When does fragmentation of breeding habitat affect population survival? Ecological Modelling 105: 273-292.

Gaplan. 1986. Atlas de Santa Catarina. Florianópolis, Aerofoto Cruzeiro.

Gascon, C.; T.E. Lovejoy; R.O. Bierregaard Jr; J.R. Malcolm; P.C. Stouffer; H.L. Vasconcelos; W.F. Laurance; B. Zimmerman; M. Tocher \& S. Borges. 1999. Matrix habitat and species richness in tropical forest remnants. Biological Conservation 91: 223-229.

Ghizoni-Jr, I. V. \& M.A.G. Azevedo. 2006. Composição de bandos mistos de aves florestais de sub-bosque em áreas de encosta e planície da Floresta Atlântica de Santa Catarina, sul do Brasil. Biotemas 19 (2): 47-57.

Hawrot, R.Y. \& G.J. Niemi. 1996. Effects of edge type and patch shape on avian communities in a mixed conifer-hardwood forest. The Auk 113: 586-598.

HutTo, R.L. 1994. The composition and social organisation of mixed-species flocks in a tropical deciduous forest in western Mexico. The Condor 96: 105-118.

Jullien, M. \& J.-M. Thiollay. 1998. Multi-species territoriality and dynamic of Neotropical forest understory bird flocks. Journal of Animal Ecology 67: 227-252.

KLEIN, R.M. 1980. Ecologia da flora e vegetação do Vale do Itajaí. Sellowia 32: 165-389.

Kotagama, S.W. \& E. Goodale. 2004. The composition and spacial organization of mixed-species flocks in a Sri Lankan rainforest. Forkail 20: 63-70.

LeE, T.M.; M.C.K. SoH; N. SodHI; L.P. КoH \& S.L.-H. Lim. 2005. Effects of habitat disturbance on mixed species bird flocks in a tropical sub-montane rainforest. Biological Conservation 122: 193-204.

Maldonado-Coelho, M. \& M.Â. Marini. 2000.Effects of Forest fragment size and successional stage of mixed-species bird flocks in southeastern Brazil. Condor 102: 585-594.

Maldonado-Coelho, M. \& M.Â. Marini. 2003. Composição de bandos mistos de aves em fragmentos de Mata Atlântica no sudeste do Brasil. Papéis Avulsos de Zoologia 43 (3): 3154.

Maldonado-Coelho, M. \& M.Â. Marini. 2004. Mixed-species bird flocks from Brazilian Atlantic Forest: the effects of forest fragmentation and seasonality on their size, richness and stability. Biological Conservation 116: 19-26.

PILlar, V.D. 1999. The bootstrapped ordination reexamined. Journal of Vegetation Science 10: 895-902.

PILLAR, V.D. 2006. MULTIV: Multivariate Exploratory Analysis, Randomization Testing and Bootstrap Resampling. PortoAlegre, Departamento de Ecologia, User's Guide v. 2.4, available online at: http://ecoqua.ecologia.ufrgs.br

Pires, A.S.; F.A.S. Fernandez \& C.S. Barros. 2006. Vivendo em um mundo em pedaços: efeitos da fragmentação florestal sobre comunidades e populações animais, p. 231-260. In: C.F.D. Rocha; H.G. Bergallo; M. Van Sluys \& M.A.S. Alves (Eds). Biologia da Conservação: essências. São Carlos, RiMa, 582p.

Pomara, L.Y.; R.J. Cooper \& L.J. Petit. 2003. Mixed-species flocking and foraging behavior of four Neotropical warblers in Panamanian shade coffee fields and forests. The Auk 120 (4): 1000-1012.

Rajão, H. \& R. Cerqueira. 2006. Distribuição altitudinal e simpatria das aves do gênero Drymophila Swainson (Passeriformes, Thamnophilidae) na Mata Atlântica. Revista Brasileira de Zoologia 23 (3): 597-607.

R Development Core Team. 2007. R: A language and environment for statistical computing. Vienna, R Foundation for Statistical Computing, available online at: www.R-project.org [Accessed: 04/XI/2008]

Rodrigues, M.; S.M.R. Alvares \& C.G. MAchado. 1994. Foraging behavior of the white-collared foliage-gleaner (Anabazenops fuscus), a bamboo specialist. Ornitologia Neotropical 5: 6567. 
Sekercioglu, C. H.; P. R. Ehrlich; G. C. Daily; D. Aygen; D. Goehring \& R. F. SANDI. 2002. Disappearance of insectivorous birds from tropical forest fragments. Proceedings of the National Academy of Sciences 99: 263-267.

SICK, H. 1997. Ornitologia Brasileira. Rio de Janeiro, Nova Fronteira, 912p.

Sridhar, H. \& K. Sankar. 2008. Effects of habitat degradation on mixed-species bird flocks in Indian rain forests. Journal of Tropical Ecology 24: 135-147.

SтотZ, D.F. 1993.Geographic variation in species composition of mixed species flocks in lowland humid forest in Brazil. Papéis Avulsos de Zoologia 38 (4): 61-75.

Stotz, D.F.; J.W. Fitzpatrick; T.A. Parker III \& D.K. Moskovits. 1996. Neotropical birds: ecology e conservation. Chicago, University of Chicago Press, 478p.
Stouffer, P.C. \& R.O. BierregaArd Jr. 1995. Use of Amazonian forest fragments by understory insectivorous birds. Ecology 76 (8): 2429-2445.

Telleria, J.L.; E.Virgós; R. Carbonell; J. Pérez-Tris \& T. Santos. 2001. Behavioural responses to changing landscapes: flocks structure and anti-predator strategies of tits wintering in fragmented forest. Oikos 95: 253-264.

Van Houtan, K.S.; S.L. Pimm; R.O. Bierregaard Jr; T.E. Lovejoy \& P.C. Stouffer. 2006. Local extinctions in flocking birds in Amazonian forest fragments. Evolutionary Ecology Research 8: 129-148.

Villard, M-A.; M.K.Trzcinski \& G. Merrian. 1999. Fragmentation effects on forest birds: relative influence of woodland cover and configuration on landscape occupancy. Conservation Biology 13 (4): 774-783.

Submitted: 04.VIII.2008; Accepted: 08.IX.2009.

Editorial responsibility: Luciano M. Verdade 